
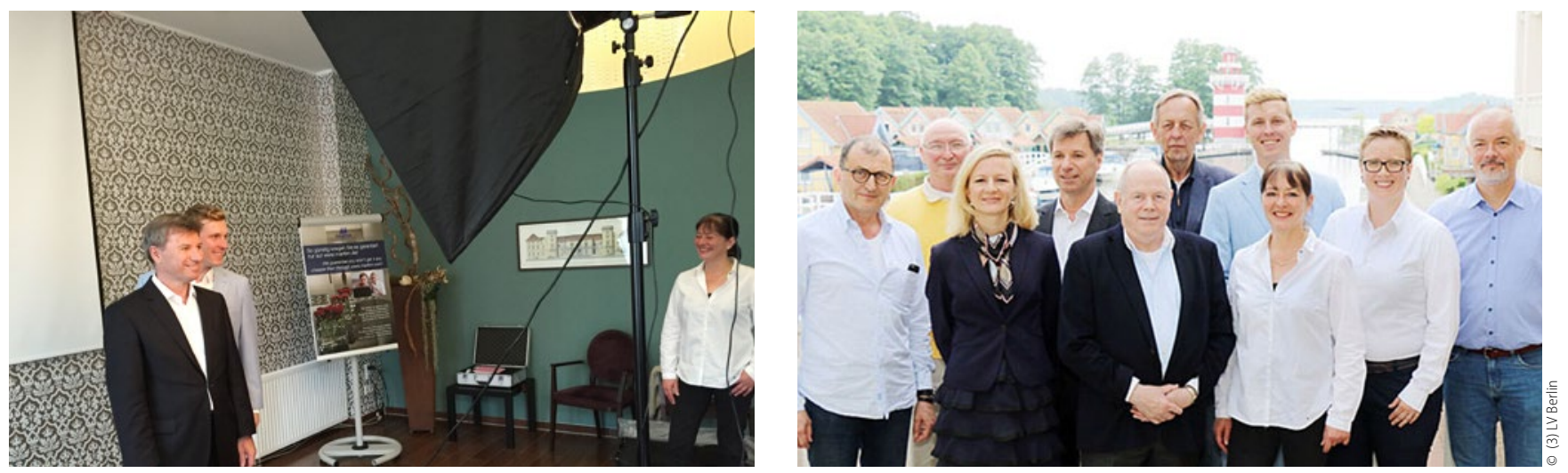

Klausurtagung des FVDZ-Landesverbandes Berlin

\title{
Malerisch und inhaltlich
}

Klausurtagung mal anders: Die Landesvorsitzende des Freien Verbandes Deutscher Zahnärzte in Berlin, Thekla Wandelt, lud die Mitglieder ihres Landesverbandes ins malerische Rheinsberg ein. Dieser staatlich anerkannte Erholungsort, berühmt geworden unter anderem durch Kurt Tucholskys Werk "Rheinsberg: Ein Bilderbuch für Verliebte“, bot den optimalen Rahmen für das Treffen.

Während der Freitagabend noch mit einem gemeinsamen Abendessen und anschließendem Spaziergang am Rheinsberger See gemütlich ausklang, stand am nächsten Morgen bei der Versammlung die Nachlese der KZV-Wahl im Fokus. Die insgesamt 222 Wählerstimmen erbrachten dem Freien Verband sechs Sitze in der Vertreterversammlung der KZV Berlin. Dieses Wahlergebnis, die Wahlvorbereitung an sich und die Möglichkeiten, die sich daraus ergeben, wurden ausgiebig diskutiert und erörtert.

Da in Berlin dieses Jahr auch noch die Wahl der Delegiertenversammlung für die Kammer stattfindet, wurde die Planung dieser Wahl inhaltlich ausgefüllt. Passend dazu hatte der Landesverband einen professionellen Fotografen engagiert, der Portrait- und Gruppenaufnahmen schoss. Diese werden für zukünftige Publikationen dringend gebraucht, und das Shooting brachte eine willkommene Abwechslung. Im Laufe des Tages wurden weitere Themen diskutiert, zum Beispiel die steigende

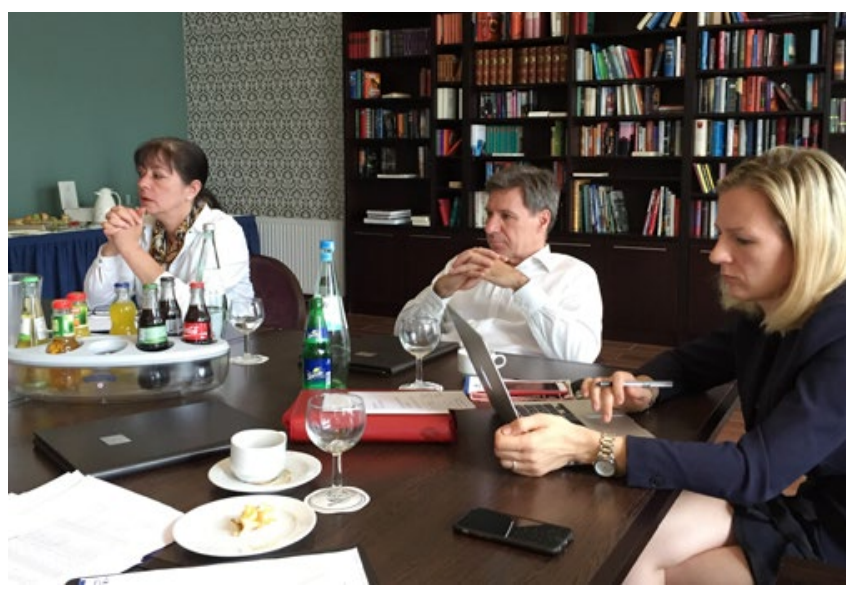

Anzahl der Medizinischen Versorgungszentren, die Stellung der angestellten Zahnärzte im FVDZ und die Betreuung der Berliner Studentenschaft.

Diese Klausurtagung war sehr ergebnisreich, zahlreiche anregende Gespräche wurden geführt, es wurde viel gelacht und am Ende waren alle Teilnehmer sehr zufrieden. Wir danken unserer Landesvorsitzenden Thekla Wandelt und ihren Stellvertretern für die Organisation. Voll motiviert können wir nun die anstehenden Aufgaben gemeinsam angehen.

Dr. Jana Lo Scalzo

\section{Save the dates}

Im September lädt der FVDZ-Landesverband Berlin seine Mitglieder herzlich zur Landesversammlung ein. Am Sonnabend, 24.09.2016, ab $10 \mathrm{Uhr}$ gibt es im Löwenbräu am Gendarmenmarkt, Leipziger Str. 65, 10117 Berlin, wieder Gelegenheit zum Austausch unter Kollegen. Der Landesverband freut sich besonders auch über die Teilnahme der jungen Kolleginnen und Kollegen sowie der studentischen Mitglieder.

Nach der Wahl ist vor der Wahl: Nachdem im Frühjahr schon die Wahl für die Delegiertenversammlung der KZV stattfand, steht der Wahlzeitraum für die Delegiertenversammlung der Zahnärztekammer Berlin fest. Im Zeitraum vom 17.11. bis zum 1.12.2016 (bis $15 \mathrm{Uhr}$ ) können alle Kammerangehörigen (Aus- und Weiterbildungsassistenten, angestellte Zahnärzte, niedergelassene Zahnärzte, Rentner) von ihrem Recht per Briefwahl Gebrauch machen. Die Wahlschreiben werden wie immer von der Kammer an die Wahlberechtigten rechtzeitig ausgesendet.

Der FVDZ-Landesverband fordert ausdrücklich alle Mitglieder auf, sich an dieser Wahl zu beteiligen. Nur mit den Stimmen aller FVDZ-Mitglieder kann sich der Verband auch in Zukunft für seine gemeinsamen Ziele stark machen.

Dr. Jana Lo Scalzo 